\title{
Adverse events in emergency oncological spine surgery: a prospective analysis
}

\author{
Clinical article
}

\author{
Nicolas Dea, M.D., ${ }^{1}$ Anne Versteeg, B.Sc., ${ }^{2}$ Charles Fisher, M.D., M.H.Sc., ${ }^{1}$ \\ Adrienne Kelly, M.D., ${ }^{1}$ Dennis Hartig, M.D., ${ }^{1}$ Michael Boyd, M.D., 1 \\ Scott Paquette, M.D., ${ }^{1}$ Brian K. Kwon, M.D., Ph.D., ${ }^{1}$ Marcel Dvorak, M.D., ${ }^{1}$ \\ And John STreet, M.D., PH.D. ${ }^{1}$
}

${ }^{I}$ Combined Neurosurgical and Orthopaedic Spine Program, University of British Columbia, Vancouver General Hospital, Vancouver, British Columbia, Canada; and ${ }^{2}$ Department of Orthopaedic Surgery, University Medical Center Utrecht, The Netherlands

\begin{abstract}
Object. Most descriptions of spine surgery morbidity and mortality in the literature are retrospective. Emerging prospective analyses of adverse events (AEs) demonstrate significantly higher rates, suggesting underreporting in retrospective and prospective studies that do not include AEs as a targeted outcome. Emergency oncological spine surgeries are generally palliative to reduce pain and improve patients' neurology and health-related quality of life. In individuals with limited life expectancy, AEs can have catastrophic implications; therefore, an accurate AE incidence must be considered in the surgical decision-making process. The purpose of this study was to determine the true incidence of AEs associated with emergency oncological spine surgery.

Methods. The authors carried out a prospective cohort study in a quaternary care referral center that included consecutive patients admitted between January 1, 2009, and December 31, 2012. Inclusion criteria were all patients undergoing emergency surgery for metastatic spine disease. AE data were reported and collected on standardized AE forms (Spine AdVerse Events Severity System, version 2 [SAVES V2] forms) at weekly dedicated morbidity and mortality rounds attended by attending surgeons, residents, fellows, and nursing staff.

Results. A total of 101 patients (50 males, 51 females) met the inclusion criteria and had complete data. Seventysix patients $(76.2 \%)$ had at least $1 \mathrm{AE}$, and 11 patients (10.9\%) died during their admission. Intraoperative surgical AEs were observed in $32 \%$ of patients (9.9\% incidental durotomy, $16.8 \%$ blood loss $>2 \mathrm{~L}$ ). Transient neurological deterioration occurred in 6 patients $(5.9 \%)$. Infectious complications in this patient population were significant (surgical site $6 \%$, other $50.5 \%$ ). Delirium complicated the postoperative period in $20.8 \%$ of cases.

Conclusions. When evaluated in a rigorous prospective manner, metastatic spine surgery is associated with a higher morbidity rate than previously reported. This AE incidence must be considered by the patient, oncologist, and surgeon to determine appropriate management and preventative strategies to reduce AEs in this fragile patient population.
\end{abstract}

(http://thejns.org/doi/abs/10.3171/2014.7.SPINE131007)

\section{KeY WORDS • adverse events - $\quad$ spinal metastasis $\quad \bullet \quad$ spine surgery $\quad$ oncology}

$\mathrm{H}$ ISTORICALLY, most reports on adverse events (AEs) in the literature are retrospective in nature or are from studies that do not include AE compilation as a primary outcome. Emerging prospective studies with AEs as the targeted outcome ${ }^{3,17}$ suggest that when prospectively collected, AE rates are significantly higher than previously reported. This discrepancy is multifactorial but explained primarily by complication studies that relied on hospital-based administrative databases that lack sufficient detail and are populated retrospectively.

Abbreviations used in this paper: $\mathrm{AE}=$ adverse event; $\mathrm{ASIA}=$ American Spinal Injury Association; CCI = Charlson Comorbidity Index; SAVES V2 = Spine AdVerse Events Severity System, version 2 .
Street et al. reported on 942 consecutive patients admitted to a quaternary care referral center undergoing spinal surgery and found that $87 \%$ had at least one documented AE. ${ }^{17}$ The patient population represented all admissions undergoing any type of spine surgery, either emergency or elective. Campbell et al. ${ }^{3}$ prospectively studied 128 patients undergoing thoracic and/or lumbar spine surgery at the neurosurgical spine unit of a university hospital and documented AEs occurring within the first 30 days of the operative procedure. They found that $59.4 \%$ of the patients experienced at least one AE. ${ }^{3}$

This article contains some figures that are displayed in color online but in black-and-white in the print edition. 


\section{Adverse events in emergency oncological spine surgery}

Fourteen percent of cancer patients will develop symptomatic spine metastasis, and this number is likely to increase as systemic treatments continue to improve. Surgery has become an evidence-based and integral part of the treatment paradigm for these patients, with indications including high-grade epidural spinal cord compression, neurological compromise, spinal instability, and tumor progression after radiation therapy., ${ }^{2,5,11,19}$ The goals of surgical management are preservation of neurological function, ensuring spinal stability, and achieving local tumor control. Surgical intervention is not without risk, however, and the benefits must be weighed against the mortality and morbidity profiles of the procedure. AE occurrence has the potential to be catastrophic in this fragile oncological population for whom quality of life is so paramount in their remaining time. The societal costs of these AEs for this palliative intervention is also an important consideration. 6.18

The primary purpose of this study was to determine the true incidence of AEs in this specific population of patients undergoing emergency spine surgery for metastatic epidural spine disease and to compare the results of an AE-targeted prospective study to the best available literature. We hypothesized that $\mathrm{AE}$ rates would be higher when collected prospectively compared to retrospectively. The secondary objectives of this study were the evaluation of the impact of AEs on the length of stay and overall patient survival.

\section{Methods}

\section{Design}

This study was a prospective observational analysis of consecutive patients admitted to our academic quaternary referral center over a 4-year period between January 1, 2009, and December 31, 2012. All patients discharged within this 4-year period with a primary or secondary diagnosis of metastatic spine disease who required an emergency surgery were included. No specific time frame was defined to meet the emergency criteria. Rather, emergency surgery was defined as surgery on patients admitted through the emergency department or seen in an outpatient clinic emergently for acute symptom onset who required immediate surgical attention either for neural structure compression or instability. Intradural metastases and primary bone tumors were excluded. Ethics committee approval was obtained according to the institutional standards.

\section{Data Collection}

Every patient's case was discussed at weekly morbidity and mortality rounds attended by all attending surgeons, residents, fellows, and nursing staff. All the AEs encountered in the previous week are discussed and coded during these rounds. The statuses of each in-hospital patient and all patients discharged since the last meeting were reviewed by all attendees. In addition, a dedicated research nurse completed daily rounds on every in-hospital patients with the purpose of identifying and documenting all AEs. In an effort to ensure that all AEs were identi- fied, patients were also discussed at daily ward rounds, where any new AE was also recorded, and each patient was again discussed with the discharge coordinator at the time of discharge. If a patient was discharged without a completed Spine AdVerse Events Severity System, version 2 (SAVES V2) ${ }^{14}$ form, the form was returned to the responsible attending surgeon within 1 week of discharge for completion. These multiple quality-assurance checks were developed to ensure maximal accuracy of our AE recording system and to ensure that no AEs were missed. All AEs were prospectively recorded using the SAVES V2 collection form, ${ }^{13,14}$ which was previously tested for reliability and validity in both elective degenerative and emergency traumatic populations. The SAVES form contains a list of common intra- and postoperative AEs and was developed specifically for spinal surgery. The occurrence of any one discrete AE in this study did not necessarily imply a measurable adverse consequence. This form was used to ensure accurate and reliable AE data identification and collection, which is critical to the development of clinical care guidelines and standards, to resource and funding allocation, and to facilitate meaningful multicenter and multidisciplinary collaboration.

\section{Data}

Demographic data, including age, sex, primary tumor histology, and comorbidities (Charlson Comorbidity Index $[\mathrm{CCI}]$ ), were collected from a local prospective spine database. Admission motor score was calculated by the surgical team using the International Standards for Neurological and Functional Classification of Spinal Cord Injury by the American Spinal Injury Association (ASIA). The type of surgical intervention was not standardized and was left to the discretion of the treating surgeon based on the specific clinical scenario for each patient. AEs occurring after discharge were collected again prospectively. Because this is the only referral center for spine-related cancer treatment within the region, all patients requiring readmission were admitted back to our institution.

\section{Statistical Analysis}

Statistical analysis was conducted using logistic regression and forward selection models to define the impact of these variables on the overall AE rate. All AE analyses were adjusted for age. The Hosmer-Lemeshow goodness-of-fit test for logistic regression models was performed to confirm adequate model fit on the forward selection models. We also performed length of stay analyses and generated Kaplan-Meier survival curves.

\section{Results}

A total of 101 patients met our inclusion criteria, including 50 males $(49.5 \%)$ and 51 females (50.5\%). Complete data were available for analysis in all 101 patients $(100 \%)$. The median age at admission was 62 years (range 33-85 years). The mean CCI score ${ }^{4}$ was 8 . The primary tumor distribution and patient characteristics are shown in Table 1. The main clinical presentation was myelop- 
N. Dea et al.

TABLE 1: Characteristics of 101 patients undergoing emergency oncological spine surgery

\begin{tabular}{lc}
\hline \multicolumn{1}{c}{ Factor } & Value \\
\hline demographics & \\
male sex (\%) & 49.5 \\
median age (yrs) & 61.9 \\
mean CCl score & 8 \\
primary tumor (\%) & \\
breast & 21.8 \\
non-small cell lung & 19.8 \\
kidney & 16.8 \\
colorectal & 10.9 \\
prostate & 6.9 \\
lymphoma & 5.0 \\
other & 18.8 \\
clinical presentation (\%) & \\
mechanical pain & 37.6 \\
radiculopathy & 23.8 \\
myelopathy & 38.6 \\
preop radiotherapy & 19.8 \\
ASIA impairment grade (\%) & \\
A & 2.0 \\
B & 1.0 \\
C & 8.9 \\
D & 46.5 \\
E & \\
\hline & \\
\hline &
\end{tabular}

athy in 39 patients (38.6\%), mechanical pain in 38 patients $(37.6 \%)$, and radiculopathy in 24 patients $(23.8 \%)$. Forty-four patients $(43.6 \%)$ had visceral metastases on initial presentation. The mean motor score was 88 (range $0-100)$, and 53 patients $(52.5 \%)$ had motor function intact on admission.

Spinal metastases were distributed as follows: cervical spine in 16 cases $(15.8 \%)$, thoracic in $63(62.3 \%)$, lumbar in $20(19.8 \%)$, and sacral in $2(2 \%)$. The mean time from admission to surgery was 78 hours, and the mean operating time was 4.6 hours. The types of surgical approaches are listed in Table 2.

Seventy-six patients (76.2\%) had at least 1 AE during their admission (Table 3). There was a mean of 1.8 AEs per patient. One-quarter of our population $(n=27)$ experienced 3 or more AEs. There were several factors that significantly influenced the total number of AEs in the forward selection model. These included the time between admission and surgery $(\mathrm{p}=0.002)$, surgeon load $(p=0.009)$, and the mode of presentation. Surgeon load was defined as the number of cases a specific surgeon performed during the study period, and the results demonstrated that patients operated on by a surgeon who performed more of these procedures had fewer overall AEs. Also, patients who presented with myelopathy had more AEs than patients who presented with only radicular symptoms, and the latter group suffered more complications than patients without neurological deficit who pre-
TABLE 2: Surgical approach by level

\begin{tabular}{cc}
\hline \multicolumn{1}{c}{ Factor } & No. of Cases \\
\hline cervical & 18 \\
posterior & 7 \\
anterior & 7 \\
anterior/posterior & 4 \\
thoracolumbar & 83 \\
posterolateral vertebrectomy & 24 \\
decompression and fusion & 50 \\
decompression only & 6 \\
fusion only & 3 \\
\hline
\end{tabular}

sented with mechanical pain only $(p=0.001)$. Interestingly, neither the CCI score $(\mathrm{p}=0.104)$ nor the motor score on admission $(\mathrm{p}=0.175)$ reached statistical significance as factors influencing the total rate of AEs in multivariate analysis.

Thirty-two percent of the emergency oncology patients in our study suffered from at least 1 intraoperative $\mathrm{AE}$; the most common was significant blood loss (>2 L) in 17 patients $(16.8 \%)$. An iatrogenic dural tear occurred in 10 patients $(9.9 \%)$, and malposition of instrumentation

TABLE 3: Summary of AEs in 101 patients

\begin{tabular}{lc}
\hline \multicolumn{1}{c}{ Variable } & \% of Patients* \\
\hline AEs & 76.2 \\
any AEs & 1.8 \\
mean no. AEs/patient & 10.9 \\
in-hospital death & 31.7 \\
intraop AEs, overall & 16.8 \\
blood loss >2 L & 9.9 \\
iatrogenic dural tear & 5.9 \\
revision of instrumentation & 2.0 \\
nerve root injury & 1.0 \\
cardiac event & 66.3 \\
postop AEs, overall & 20.8 \\
delirium & 5.9 \\
transient neurological deterioration & 4.0 \\
pressure sores & 4.0 \\
deep vein thrombosis & 2.0 \\
early construct failure & 11.0 \\
electrolyte imbalance & 4.0 \\
arrhythmia & 5.0 \\
dysphagia & 34.7 \\
infection & 11.9 \\
urinary tract infection & 3.9 \\
pneumonia & 3.9 \\
systemic sepsis & 2.0 \\
deep wound infection & \\
superficial wound infection & \\
\hline
\end{tabular}

* Values are the percentage of 101 patients unless otherwise stated. 
requiring revision occurred in 6 patients $(5.9 \%)$. One patient (1\%) had an intraoperative cardiac event, and 2 had an unplanned nerve root injury (2\%). The length of surgery $(p=0.029)$ was the strongest predictor of intraoperative AEs.

Two-thirds of our patients developed postoperative complications. Two factors had a statistically significant unfavorable impact on these complications in the forward selection models: longer duration of time from admission to surgery $(\mathrm{p}=0.021)$ and age at admission $(\mathrm{p}=0.035)$. Twenty-one patients (20.8\%) developed delirium, making it one of the most common AEs complicating the postoperative course of our study population. Delirium was more common in male patients $(\mathrm{p}=0.031)$. Transient neurological deterioration occurred in 6 patients (5.9\%). No patients suffered from permanent neurological deterioration. Infectious complications occurred in a significant number of patients. Thirty-five (34.7\%) had a urinary tract infection, 12 (11.9\%) developed pneumonia, and 4 patients (3.9\%) developed systemic sepsis. Surgical site infection was documented in 6 patients: $4(3.9 \%)$ developed deep wound infections and $2(2.0 \%)$ developed superficial infections. Twenty patients (19.8\%) received preoperative radiation therapy and subsequently had surgery because of radiation treatment failure; however, neither pre- nor postoperative radiation therapy influenced the rate of wound complications in our study $(p=0.968)$. The deep wound infection rate was increased by the presence of an iatrogenic dural tear $(p=0.024)$ and if the patient required another surgery during the same admission $(\mathrm{p}=$ 0.002 ). Three patients required revision surgery during their index admission: 2 for wound infection and 1 for hardware revision. Four patients (4.0\%) developed pressure sores during their hospitalization. The presence of pressure sores was influenced by the motor score on admission $(p=0.031)$ and the time from admission to surgery $(p=0.037)$. Four subjects had a deep vein thrombosis (4\%), and 2 had an early construct failure (2\%). Other AEs, which by themselves did not have significant clinical impact but are worth mentioning for completeness, were electrolyte imbalance (11\%), hematological complication (2\%), transient cardiac arrhythmia (4\%), and short-lived postoperative dysphagia (5\%).

The median length of stay was 21 days (range 4-100 days). The presence of any AE clearly affected the length of stay ( $p<0.0001)$. As we would expect, the need for a second operation was also significant in the multivariate analysis $(p=0.006)$ for increased length of stay. Motor score on admission $(\mathrm{p}<0.0001)$ and postoperative deep venous thrombosis $(\mathrm{p}=0.0013)$ were also significant in the multivariate analysis for increased length of stay. Eight patients (8\%) required readmission for an AE: 4 for a deep wound infection, 2 for failure of instrumentation, and 2 for pain management.

Twenty-four patients $(23.8 \%)$ were still alive at the last follow-up. The median survival after spinal surgery was 8.4 months; the Kaplan-Meier survival curve is shown in Fig. 1. There were 11 in-hospital deaths (10.9\%). These were not necessarily related to postoperative AEs but rather to a more palliative orientation and withdrawal of aggressive treatment according to patient and family

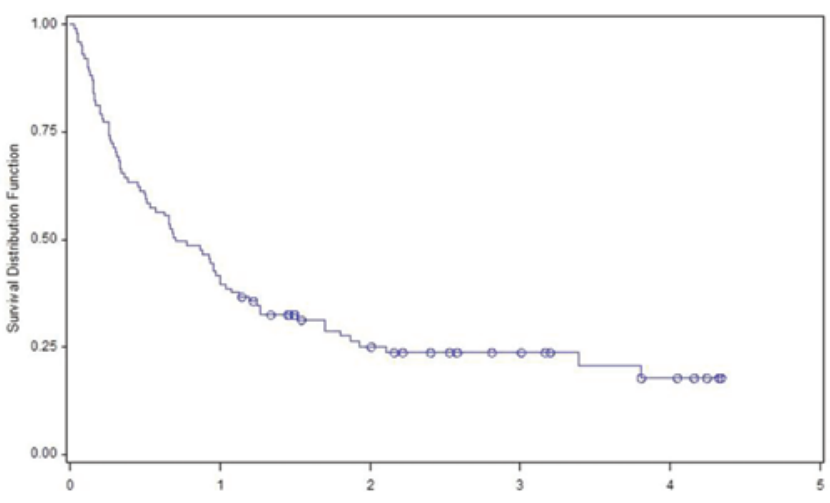

FIG. 1. Kaplan-Meier survival curve. The line shows the product-limit estimate curve. Censored observations are indicated with circles.

wishes. Overall survival was statistically influenced by the surgery duration, number of AEs, male sex, primary disease, surgeon load, and the initial ASIA impairment scale score. In addition, overall survival was also influenced by the presence of postoperative delirium. Yet, in our study, overall survival was not influenced by the CCI score or the extent of disease.

\section{Discussion}

Using a detailed prospective data collection method and the validated AE data collection form, SAVES V2, we examined 101 consecutive patients undergoing emergency surgery for spinal metastases and found an overall $\mathrm{AE}$ rate of $76.2 \%$. This is significantly higher than previously reported in the spine literature. Historically, the complication rates range from $15 \%$ to $39 \%$. $^{9,10,12,15}$ Wise et al. conducted a retrospective analysis on complications, survival rates, and risk factors of surgery for metastatic disease of the spine and found a complication rate of $25 \%$ in 80 patients. ${ }^{20}$ The likelihood of having a complication in their study was strongly related to the Harrington classification of spinal metastatic disease, presence of neurological deficits, and preoperative radiation therapy. Although the presence of myelopathy or radiculopathy was associated with an increased overall AE rate, we did not find similar a association between the preoperative motor score and $\mathrm{AE}$ rate. Also, preoperative radiation therapy did not seem to have a significant impact on wound complications in our study. This is in contrast to what was previously published on this subject.? However, their study was a retrospective chart review including patients from 1970 through 1996 who underwent surgical decompression for spinal cord compression after irradiation. $.^{20} \mathrm{Ad}-$ vancements in radiation treatments, surgical techniques, and postoperative care might explain the difference in the influence of radiation on wound complications. Another reason for this discrepancy may be that our study was underpowered to assess this outcome.

Wang et al. ${ }^{19}$ reported a combined major complication and reoperation rate of $25.3 \%$ in patients undergoing single-stage posterolateral decompression for epidural metastatic disease. Like most other studies, this was a retrospective analysis investigating a specific procedure. In a retrospective study of 200 patients surgically treated for 
spine metastases, Arrigo et al. found that $34 \%$ of their patients had at least one AE. ${ }^{1}$ The CCI score ${ }^{4}$ was the most significant predictor of AE occurrence in their study. We did not adjust for oncological factors in our statistical analysis, which may explain why we did not reproduce these findings. Similarly Arrigo et al. and Shehadi et al. did not find a positive correlation between perioperative radiation therapy and wound complications. ${ }^{1,15}$

Our total AE rates are not significantly different from those reported by Street et al.. ${ }^{17}$ who published a study of 942 consecutive patients undergoing spinal surgery for all indications. This may suggest that, despite having metastatic disease, this population is not significantly more prone to developing AEs. Interestingly, another study by Street et al. ${ }^{16}$ at the same institution reported AEs in 47\% of the patients who underwent a posterolateral vertebrectomy for spinal metastatic disease. This retrospectively collected AE rate was significantly lower than the rate reported in their prospective study, even though both were conducted at the same spine unit, once again reinforcing the superiority of prospective AE collection.

Accurate AE rates are important to both patients and physicians. They allow the patient to have all the necessary information to provide more precise informed operative consent. For the surgeon, it provides quality assurance and a useful basis to develop preventive and risk reduction measures with the ultimate goal of improved patient care. It also assists in the decision-making process and improves the treatment algorithm employed by the multidisciplinary team of physicians caring for these patients by confronting a known risk profile to an expected benefit. Having a disease-specific AE profile is even more useful because it allows physicians to apply it to real-life, day-to-day clinical scenarios.

AEs are inevitable in any type of surgical intervention. To be able to recognize them in a timely fashion and react accordingly is certainly very important. More important is the use of valid AE data to initiate preventative strategies. As expected based on other prospective studies on AEs, our AE rate was higher than that reported in retrospective studies. This may be due to the prospective nature of the data collection as well as the rigorous methodology we employed. In addition, we collected all potential AEs, not only the ones considered medically relevant, which may be different from the approach used in previous publications and likely contributes to the higher $\mathrm{AE}$ rate. This resulted in the recording of AEs that are not necessarily direct postoperative complications but rather secondary to the primary diagnosis. Recording all of these AEs provides a more accurate picture of what can be expected during the admission of a patient with a spinal metastasis, and it also contributes to surprisingly high rates of AEs. The assumption that the clinical impact of some AEs may be of questionable significance and therefore may be ignored has never been studied before. It is imprudent to assume that there is no effect of an AE; indeed, in combination with other AEs, it can potentially influence patient outcome. Furthermore, there is the perception that minor AEs are insignificant, but recent data suggest that they are responsible for one-third of total $\mathrm{AE}$ costs. ${ }^{8}$ Increased length of stay contributes to increased cost and negatively impacts patient quality of life. It is therefore important to limit the occurrence of even the most benign AEs.

\section{Conclusions}

To the best of our knowledge, this study is the first detailed, prospective analysis using a validated data collection instrument specifically looking at AEs in an emergency oncological setting. The results revealed that when evaluated in a rigorous prospective manner, metastatic spine surgery is associated with a higher morbidity than previously reported. This AE incidence must be considered by the patient, oncologist, and surgeon in determining appropriate management and preventative strategies to reduce AEs in this fragile patient population. Quality of life and health economic impact studies related to AEs in patients with metastatic spine disease are lacking and are much needed to improve patient outcomes.

\section{Disclosure}

Dr. Dvorak holds a patent with and works as a consultant for Medtronic. He receives research support from AOSpine, Synthes, Medtronic, and DePuy. Dr. Fisher serves as a consultant for Medtronic and NuVasive.

Author contributions to the study and manuscript preparation include the following. Conception and design: Dea, Fisher, Street. Acquisition of data: Dea, Versteeg, Hartig, Boyd, Paquette, Kwon, Dvorak, Street. Analysis and interpretation of data: Dea, Versteeg, Street. Drafting the article: Dea, Versteeg. Critically revising the article: all authors. Reviewed submitted version of manuscript: all authors. Approved the final version of the manuscript on behalf of all authors: Dea. Administrative/technical/material support: Fisher, Paquette, Kwon, Dvorak, Street. Study supervision: Dea.

\section{References}

1. Arrigo RT, Kalanithi P, Cheng I, Alamin T, Carragee EJ, Mindea SA, et al: Charlson score is a robust predictor of 30day complications following spinal metastasis surgery. Spine (Phila Pa 1976) 36:E1274-E1280, 2011

2. Bilsky MH, Boland P, Lis E, Raizer JJ, Healey JH: Singlestage posterolateral transpedicle approach for spondylectomy, epidural decompression, and circumferential fusion of spinal metastases. Spine (Phila Pa 1976) 25:2240-2250, 2000

3. Campbell PG, Malone J, Yadla S, Maltenfort MG, Harrop JS, Sharan AD, et al: Early complications related to approach in thoracic and lumbar spine surgery: a single center prospective study. World Neurosurg 73:395-401, 2010

4. Charlson ME, Pompei P, Ales KL, MacKenzie CR: A new method of classifying prognostic comorbidity in longitudinal studies: development and validation. J Chronic Dis 40:373383, 1987

5. Falicov A, Fisher CG, Sparkes J, Boyd MC, Wing PC, Dvorak MF: Impact of surgical intervention on quality of life in patients with spinal metastases. Spine (Phila Pa 1976) 31:2849-2856, 2006

6. Furlan JC, Chan KK, Sandoval GA, Lam KC, Klinger CA, Patchell RA, et al: The combined use of surgery and radiotherapy to treat patients with epidural cord compression due to metastatic disease: a cost-utility analysis. Neuro Oncol 14:631-640, 2012

7. Ghogawala Z, Mansfield FL, Borges LF: Spinal radiation before surgical decompression adversely affects outcomes of surgery for symptomatic metastatic spinal cord compression. Spine (Phila Pa 1976) 26:818-824, 2001 


\section{Adverse events in emergency oncological spine surgery}

8. Hellsten EK, Hanbidge MA, Manos AN, Lewis SJ, Massicotte EM, Fehlings MG, et al: An economic evaluation of perioperative adverse events associated with spinal surgery. Spine J 13:44-53, 2013

9. Hirabayashi H, Ebara S, Kinoshita T, Yuzawa Y, Nakamura I, Takahashi J, et al: Clinical outcome and survival after palliative surgery for spinal metastases: palliative surgery in spinal metastases. Cancer 97:476-484, 2003

10. Jansson KA, Bauer HC: Survival, complications and outcome in 282 patients operated for neurological deficit due to thoracic or lumbar spinal metastases. Eur Spine J 15:196-202, 2006

11. Patchell RA, Tibbs PA, Regine WF, Payne R, Saris S, Kryscio RJ, et al: Direct decompressive surgical resection in the treatment of spinal cord compression caused by metastatic cancer: a randomised trial. Lancet 366:643-648, 2005

12. Patil CG, Lad SP, Santarelli J, Boakye M: National inpatient complications and outcomes after surgery for spinal metastasis from 1993-2002. Cancer 110:625-630, 2007

13. Rampersaud YR, Moro ERP, Neary MA, White K, Lewis SJ, Massicotte EM, et al: Intraoperative adverse events and related postoperative complications in spine surgery: implications for enhancing patient safety founded on evidence-based protocols. Spine (Phila Pa 1976) 31:1503-1510, 2006

14. Rampersaud YR, Neary MA, White K: Spine adverse events severity system: content validation and interobserver reliability assessment. Spine (Phila Pa 1976) 35:790-795, 2010

15. Shehadi JA, Sciubba DM, Suk I, Suki D, Maldaun MVC, McCutcheon IE, et al: Surgical treatment strategies and outcome in patients with breast cancer metastatic to the spine: a review of 87 patients. Eur Spine J 16:1179-1192, 2007

16. Street J, Fisher C, Sparkes J, Boyd M, Kwon B, Paquette S, et al: Single-stage posterolateral vertebrectomy for the manage- ment of metastatic disease of the thoracic and lumbar spine: a prospective study of an evolving surgical technique. J Spinal Disord Tech 20:509-520, 2007

17. Street JT, Lenehan BJ, DiPaola CP, Boyd MD, Kwon BK, Paquette SJ, et al: Morbidity and mortality of major adult spinal surgery. A prospective cohort analysis of 942 consecutive patients. Spine J 12:22-34, 2012

18. Thomas KC, Nosyk B, Fisher CG, Dvorak M, Patchell RA, Regine WF, et al: Cost-effectiveness of surgery plus radiotherapy versus radiotherapy alone for metastatic epidural spinal cord compression. Int J Radiat Oncol Biol Phys 66:12121218, 2006

19. Wang JC, Boland P, Mitra N, Yamada Y, Lis E, Stubblefield M, et al: Single-stage posterolateral transpedicular approach for resection of epidural metastatic spine tumors involving the vertebral body with circumferential reconstruction: results in 140 patients. J Neurosurg Spine 1:287-298, 2004

20. Wise JJ, Fischgrund JS, Herkowitz HN, Montgomery D, Kurz LT: Complication, survival rates, and risk factors of surgery for metastatic disease of the spine. Spine (Phila Pa 1976) 24:1943-1951, 1999

Manuscript submitted November 12, 2013.

Accepted July 10, 2014.

An abstract of this work was presented at the 2013 AANS/CNS Joint Section on Spine \& Peripheral Nerves Meeting, Phoenix, AZ, March 6-9, 2013.

Please include this information when citing this paper: published online August 22, 2014; DOI: 10.3171/2014.7.SPINE131007.

Address correspondence to: Nicolas Dea, M.D., Blusson Spinal Cord Centre, 6th Floor, 818 W. 10th Ave., Vancouver, BC V5Z 1M9, Canada. email: nicolas.dea@vch.ca. 